\title{
Dynamics of ring dark solitons in Bose-Einstein condensates and nonlinear optics
}

\author{
A.M. Kamchatnov, S.V. Korneev \\ Institute of Spectroscopy, Russian Academy of Sciences, Troitsk, Moscow Region, 142190, \\ Russia
}

\begin{abstract}
Quasiparticle approach to dynamics of dark solitons is applied to the case of ring solitons. It is shown that the energy conservation law provides the effective equations of motion of ring dark solitons for general form of the nonlinear term in the generalized nonlinear Schrödinger or Gross-Pitaevskii equation. Analytical theory is illustrated by examples of dynamics of ring solitons in light beams propagating through a photorefractive medium and in non-uniform condensates confined in axially symmetric traps. Analytical results agree very well with the results of our numerical simulations.
\end{abstract}

Keywords: dark ring soliton, Bose-Einstein condensate, nonlinear optics

\section{Introduction}

As is known, dark solitons are fundamental excitations of nonlinear media with modulationally stable background (see review articles [1, 2] and references therein). Usually their dynamics is modeled by a generalized nonlinear Schrödinger (NLS) or Gross-Pitaevskii (GP) equation with defocusing (repulsive) nonlinearity

$$
i \psi_{t}+\frac{1}{2} \Delta \psi-f\left(|\psi|^{2}\right) \psi=U(\mathbf{r}) \psi
$$

where $\psi=\psi(\mathbf{r}, t)$ is the field variable which interpretation depends of the physical system under consideration. For example, in case of atomic Bose-Einstein condensate (BEC) it represents the order parameter ("condensate wave function") and then $\rho=|\psi|^{2}$ has a meaning of density of atoms, $\psi=\sqrt{\rho} \exp (i \phi)$, $\mathbf{u}=\nabla \phi$ is the condensate's flow velocity, and $U(\mathbf{r})$ is the external potential of forces acting on atoms (e.g., the trap potential). In nonlinear optics, the fields variable $\psi$ has a meaning of the light field strength, $|\psi|^{2}$ its intensity, $t$ plays the role of the coordinate along the light beam, $\mathbf{r}$ is the radius-vector in transverse directions, and $U(\mathbf{r})$ can be related with non-uniformity of a refractive index.

Email addresses: kamch@isan.troitsk.ru (A.M. Kamchatnov), svyatoslav.korneev@gmail.com (S.V. Korneev) 
In the most common case the function

$$
f\left(|\psi|^{2}\right)=|\psi|^{2}
$$

corresponds to a repulsive interatomic interaction in BEC case or a defocusing Kerr nonlinearity in nonlinear optics case. If $\psi$ depends on time and one space coordinate $x$ only and the medium is uniform $(U(\mathbf{r})=0)$, then Eq. (1) with nonlinear term (2) reduces to standard 1D NLS equation

$$
i \psi_{t}+\frac{1}{2} \psi_{x x}-|\psi|^{2} \psi=0
$$

with well-known dark soliton solution

$$
\psi=\psi_{s}(x-V t)=\left\{\sqrt{\rho_{0}-V^{2}} \tanh \left[\sqrt{\rho_{0}-V^{2}}(x-V t)\right]+i V\right\} e^{-i \rho_{0} t},
$$

where $\rho_{0}$ is the background density and $V$ the soliton's velocity. Naturally, the parameters $\rho_{0}$ and $V$ are constant here.

In many situations we can approximate locally the soliton solution of the general equation (1) the soliton solution analogous to formula (4) but now with slow dependence of $\rho_{0}$ and $V$ on space and time coordinates. For example, if a dark soliton propagates in BEC confined in a cigar-shaped trap, then its dynamics can be described in certain approximation by the following generalization of Eq. (3):

$$
i \psi_{t}+\frac{1}{2} \psi_{x x}-|\psi|^{2} \psi=U(x) \psi
$$

where $U(x)=\omega_{0}^{2} x^{2} / 2$ in case of a harmonic axial potential. As was found in [3], such a soliton oscillates in a harmonic trap with the frequency $\omega=\omega_{0} / \sqrt{2}$, and it was shown in [4] that this result can be obtained most simply if one considers a dark soliton as a quasiparticle with the energy

$$
\varepsilon(V)=\frac{4}{3}\left(\rho_{0}-V^{2}\right)^{3 / 2}
$$

which expression follows easily from the Hamiltonian form of Eq. (3). Then conservation of energy

$$
\varepsilon(V)=\text { const }
$$

combined with the Thomas-Fermi (TF) distribution of the background density

$$
\rho_{0}(x)=\mu-U(x), \quad \mu=\text { const },
$$

yields at once the equation for $V=d X / d t$, where $X(t)$ is a coordinate of "position" of the soliton:

$$
\left(\frac{d X}{d t}\right)^{2}+U(x)=\text { const. }
$$

This approach was developed in more detail in Ref. [5] where, in particular, its generality was illustrated by application to the case of nonlinearity $f(\rho)=\rho^{2}$. General formulae for the case of arbitrary function $f(\rho)$ (provided that the 
condition of modulation stability of the background is satisfied) were derived later in Ref. 6]. It is clear that this approach is correct as long as width $1 / \sqrt{\rho_{0}-V^{2}}$ of the soliton is much less than a characteristic length at which the background parameters change considerably so that a "collective" coordinate $X(t)$ is a well-defined variable.

This approach admits also the form of Hamilton equations which describe dynamics of the soliton quasiparticle. Indeed, as was shown in [7] (see also [5]), the momentum of the soliton (4) is equal to

$$
p=-2 V \sqrt{\rho_{0}-V^{2}}+2 \rho_{0} \arctan \left(\sqrt{\rho_{0}-V^{2}} / V\right)
$$

and the equation

$$
\frac{\partial \varepsilon}{\partial p}=V=\frac{d X}{d t}
$$

holds which evidently is one of the Hamilton equations for the quasiparticle with the Hamiltonian (6). In case of the dark soliton moving through non-uniform $\mathrm{BEC}$ confined in a trap created by the potential $U(x)$ the density $\rho_{0}$ is a function of $x$ related with $U(x)$ by Eq. (8). Hence the Hamiltonian becomes a function of the position $X$ of the soliton, $\varepsilon(X, V) \equiv \varepsilon\left(\rho_{0}(X), V\right)$, where $\rho_{0}(X)$ is related with the potential $U(X)$ by the equation (8) with $x$ replaced by the coordinate $X$ of the soliton's position. It is clear that the equation (11) does not change provided the derivative in the left-hand side is taken for fixed $X$. Then the second Hamilton equation

$$
\frac{d p}{d t}=-\left(\frac{\partial \varepsilon(X, V)}{\partial X}\right)_{p}
$$

can be readily reduced to

$$
2 \frac{d V}{d t}=-\frac{\partial U(X)}{\partial X}
$$

that is to the Newton equation with the effective mass of the dark soliton quasiparticle equal to the doubled atomic mass as it was first discovered in [3] (see also [4, 5, 6]).

In this Letter we show that the approach of Refs [4, 5, 6] can be easily generalized to multi-dimensional situations of ring or spherical solitons. For example, if a ring dark soliton evolves in a nonlinear medium, then the speed of its radius $R(t), V=d R / d t$, depends not only on time $t$, but also on $R$. If the width of the soliton is much less than its radius, then the conservation law of the total energy in the first approximation reads

$$
2 \pi R \varepsilon\left(\rho_{0}(R), d R / d t\right)=\text { const. }
$$

Thus, we arrive at a differential equation for $R(t)$ which can be easily solved in various situations. We shall consider here by this method several typical examples. Naturally, in the simplest case of Kerr nonlinearity and uniform background our approach reproduces well-known results of Ref. [8. In actual 
optics experiments (see, e.g., 9]) on ring solitons, the photorefractive media with $f(\rho)=\rho /(1+\gamma \rho)$ were often used, and in the next section we shall consider evolution of ring dark solitons in such a medium. Evolution of ring dark solitons in BEC confined in axially symmetric traps was considered in Refs. 10, 11] in various approximations. We show in section 3 that our approach gives a very good approximation (different from ones discussed earlier) which agrees quite well with direct numerical solution of the GP equation up to the moment of decay of ring solitons to vortices due to their snake instability.

\section{Dynamics of dark ring solitons in a uniform background}

First, we shall consider the case of uniform background which density (intensity) is constant, $\rho_{0}=$ const. Although new results will be obtained for a non-Kerr nonlinearity function $f(\rho)$, we shall begin with the Kerr-like case $f(\rho)=\rho$ which illustrates very clearly the method.

\subsection{Kerr-like nonlinearity}

In case of Kerr-like nonlinearity (2), i.e. $f(\rho)=\rho$, the energy of a dark soliton per unit of its length is given by Eq. (6). Hence the total energy of a ring dark soliton with radius $R$ is equal to $2 \pi R \varepsilon$ and its conservation law yields the equation

$$
R\left(\rho_{0}-\dot{R}^{2}\right)^{3 / 2}=R_{0}\left(\rho_{0}-V_{0}^{2}\right)^{3 / 2},
$$

where $\dot{R}=d R / d t$ and $R_{0}$ and $V_{0}$ are the initial radius and velocity of the dark soliton, correspondingly. This equation was derived first in a different form in [8] with the use of perturbation theory. Its differentiation with respect to time $t$ gives the Newton-like equation

$$
\ddot{R}=\frac{1}{3 R}\left(\rho_{0}-\dot{R}^{2}\right)=\frac{R_{0}^{2 / 3}\left(\rho_{0}-V_{0}^{2}\right)}{3 R^{5 / 3}},
$$

which has been recently obtained in [12] as a particular case of equations of contour dynamics of dark solitons. Variable $R$ can be excluded from (16) with the help of Eq. 15p so that one obtains

$$
\frac{d V}{d t}=\frac{\left(\rho_{0}-V^{2}\right)^{5 / 2}}{3 R_{0}\left(\rho_{0}-V_{0}^{2}\right)^{3 / 2}},
$$

and elementary integration of this equation yields implicit dependence of $V$ on time

$$
\frac{V\left(3 \rho_{0}-2 V^{2}\right)}{\left(\rho_{0}-V^{2}\right)^{3 / 2}}=\frac{V_{0}\left(3 \rho_{0}-2 V_{0}^{2}\right)-t / R_{0}}{\left(\rho_{0}-V^{2}\right)^{3 / 2}} .
$$

This equation can be solved with respect to $V^{2}$ in a closed form (see [12]) and this analytical solution agrees very well with the results of direct numerical solution of the GP equation. Thus, interpretation of Eq. 15 as the energy conservation law permits us to reproduce very simply the known results. 


\subsection{General form of nonlinearity}

As was shown in [6], in general case the energy of a dark soliton per its unit length can be expressed as

$$
\varepsilon=\frac{1}{2} \int_{\rho_{m}}^{\rho_{0}} \frac{Q_{0}(\rho)}{\rho \sqrt{Q(\rho)}} d \rho,
$$

where

$$
\begin{gathered}
Q_{0}(\rho)=8 \rho \int_{\rho}^{\rho_{0}}\left[f\left(\rho_{0}\right)-f\left(\rho^{\prime}\right)\right] d \rho^{\prime}, \\
Q(\rho)=Q_{0}(\rho)-4 V^{2}\left(\rho_{0}-\rho\right)^{2},
\end{gathered}
$$

and the minimal value $\rho_{m}$ in the distribution of the density $\rho$ across the dark soliton dip is related with its velocity $V$ by the equation

$$
V^{2}=\frac{Q_{0}\left(\rho_{m}\right)}{4\left(\rho_{0}-\rho_{m}\right)^{2}} .
$$

Hence, $\varepsilon$ can be considered as a function of $\rho_{0}$ and $V^{2}$ and, as above, conservation of the total energy of the ring soliton reads

$$
R \varepsilon\left(\rho_{0}, \dot{R}^{2}\right)=R_{0} \varepsilon\left(\rho_{0}, V_{0}^{2}\right) .
$$

This is a differential equation for the function $R=R(t)$ with given initial conditions $R=R_{0}, V=V_{0}$ at $t=0$, and its solution describes the evolution of the ring dark soliton. To obtain more definite results, one should specify the nonlinearity function $f(\rho)$.

\subsection{Evolution of ring dark solitons in media with photorefractive nonlinearity}

In nonlinear optics experiments [9] the nonlinear effects can be modeled by the function

$$
f(\rho)=\frac{\rho}{1+\gamma \rho}
$$

which takes into account saturation of the nonlinear response, i.e. $f(\rho) \rightarrow 1 / \gamma=$ const as $\rho \rightarrow \infty$. In this case

$$
Q_{0}(\rho)=\frac{8 \rho}{\gamma^{2}}\left(\ln \frac{1+\gamma \rho_{0}}{1+\gamma \rho}+\frac{\gamma\left(\rho_{0}-\rho\right)}{1+\gamma \rho_{0}}\right) .
$$

Substitution of this expression into Eqs. 19$)$ and $(22)$ yields the function $\varepsilon\left(\rho_{0}, V^{2}\right)$ and the resulting differential equation $(23)$ can be solved numerically. Example of such a solution is shown in Fig. 1 for the choice of parameters: $\rho_{0}=1, \gamma=1$, $R_{0}=30, V_{0}=0.2$. The solid line represents the results of our approximate theory and they are compared with the exact numerical solution of the generalized NLS equation (1) with $f$ given by Eq. (24) and $U=0$; values of the dark soliton radius for different moments of time are shown by crosses. As we see, agreement is quite good. 


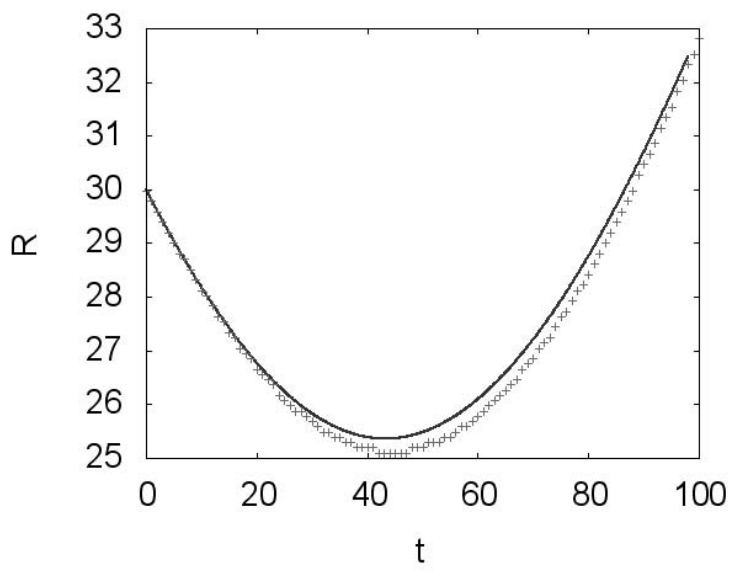

Figure 1: Dependence of the radius of the ring dark soliton on time for the case of photorefractive nonlinearity: solid line corresponds to analytical theory and crosses to numerical solution of the generalized NLS equation.

\section{Evolution of ring dark solitons in a non-uniform background}

As a typical example, we shall consider the BEC confined in an axially symmetric trap. As above, we assume that cylindrical symmetry persists during evolution so that dynamics of a ring dark soliton reduces to varying of its radius $R$ with time $t$. However, now the background density $\rho_{0}$ is not uniform and depends on the radial coordinate $r$. In the Thomas-Fermi (TF) approximation and for quadratic nonlinearity $f(\rho)=\rho$ this dependence is given by

$$
\rho_{0}(r)=\mu-U(r)
$$

and in the case of a harmonic trap $U(r)=\omega_{0}^{2} r^{2} / 2$ this dependence can be written as

$$
\rho_{0}(r)=\frac{1}{2} \omega_{0}^{2}\left(l^{2}-r^{2}\right),
$$

where $l$ is the radius of the condensate's distribution related with the chemical potential $\mu$ and the number of particles $N$ (per unit axial length) by the formulae

$$
\mu=\frac{1}{2} \omega_{0}^{2} l^{2}, \quad N=\frac{\pi}{4} \omega_{0}^{2} l^{4} .
$$

Therefore the energy conservation law now reads

$$
R \varepsilon\left(\rho_{0}(R), \dot{R}^{2}\right)=R_{0} \varepsilon\left(\rho_{0}\left(R_{0}\right), V_{0}^{2}\right) .
$$

We shall study it in some detail for a particular case of a harmonic trap when $\rho_{0}$ is given by Eq. 27) and of two-particle repulsive interatomic interaction when 
$f(\rho)=\rho$ and $\varepsilon(V)$ is given by Eq. (6). Then Eq. 29) takes the form

$$
R\left[\frac{\omega_{0}}{2}\left(l^{2}-R^{2}\right)-\left(\frac{d R}{d t}\right)^{2}\right]^{3 / 2}=R_{0}\left(\rho_{0}-V_{0}^{2}\right)^{3 / 2},
$$

where $\rho_{0}=\omega_{0}^{2}\left(l^{2}-R_{0}^{2}\right) / 2$ is the density at the initial radius $R_{0}$ of the soliton. This equation can be rewritten as

$$
\left(\frac{d R}{d t}\right)^{2}=\frac{1}{2} \omega_{0}^{2}\left(l^{2}-R^{2}\right)-\frac{R_{0}^{2 / 3}\left(\rho_{0}-V_{0}^{2}\right)}{R^{2 / 3}} .
$$

Its differentiation with respect to time $t$ gives the Newton-like equation

$$
\frac{d^{2} R}{d t^{2}}=-\frac{\omega_{0}^{2}}{2} R+\frac{R_{0}^{2 / 3}\left(\rho_{0}-V_{0}^{2}\right)}{3 R^{5 / 3}} .
$$

The first term in the right-hand side corresponds to oscillations of the soliton radius with the frequency $\omega_{0} / \sqrt{2}$, i.e. it describes the effects of non-uniformity of the condensate discovered in [3]. The second term in the right-hand side of 32 describes the effects of the curvature of the soliton and coincides with Eq. (16). In our adiabatic approximation both effects are weak and therefore they are added in the combined equation (32). Integration of this equation reduces to a quadrature

$$
\frac{\omega_{0} t}{\sqrt{2}}= \pm \int_{R_{0} / l}^{R / l} \frac{d w}{\sqrt{1-w^{2}-a w^{-2 / 3}}}, \quad a=\frac{2 R_{0}^{2 / 3}\left(\rho_{0}-V_{0}^{2}\right)}{\omega_{0}^{2} l^{8 / 3}},
$$

which actually solves the problem. In Fig. 2 we have compared this analytical solution with the exact numerical solution of Eq. (1) for the choice of the parameters $N=3486.5, \mu=1, l=50, \omega_{0}=0.03, R_{0}=10.0, V=0.229$. At the moment of time $t_{c}=79.3$ the ring soliton reaches its turning point with the maximal value of the radius $R_{\max }=37.25$; after this moment its radius decreases and, as it was already indicated in Ref. [10], the snake instability starts to develop, so that the dark soliton evolves into necklace of vortices. This process is illustrated in Fig. 3. In Fig. 2 the radius of the necklace is shown by circles and formal analytical solution corresponds here to a dashed line. As a measure of deviation of the structure from the ring soliton we have introduced the quantity

$$
\delta \rho=\sqrt{\overline{\rho^{2}}-(\bar{\rho})^{2}},
$$

where the averages $\bar{\rho}$ and $\overline{\rho^{2}}$ are taken along the circle of the minimal values of the density in the structure. Its dependence on time is shown in Fig. 4. It can be fitted by the exponential growth of $\delta \rho$ :

$$
\delta \rho \approx 8.198 \cdot 10^{-9} \exp (0.123 t)
$$

The coefficient can be represented as $8.198 \cdot 10^{-9}=0.0065 \cdot \exp (-0.123 \cdot 110)$ which corresponds to the numerically introduced disturbance about $\sim 0.01$ 


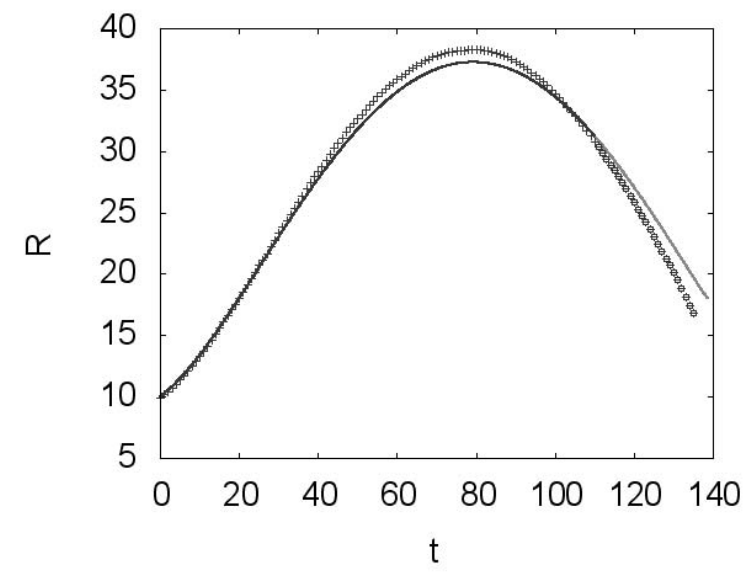

Figure 2: Dependence of the radius of the ring dark soliton on time in case of its propagation along $\mathrm{TF}$ distribution of density BEC confined in a harmonic trap: solid line corresponds to analytical theory and crosses to numerical solution of the GP equation.

which starts to grow up after the moment of time about $t \approx 110$, that is after the turning point what can be explained as a result of very slow change of parameters of the soliton in vicinity of the turning point so that the most unstable mode has enough time for its evolution without strong competition with other modes. The growth rate constant $\Gamma=0.123$ can be compared with that calculated according to the theory [13] of snake instability of plane dark solitons for given depth and background density. If we take these parameters for the moment $t=110$, when the soliton radius equals to $R=30.9$, then we get the maximal growth rate $\Gamma=0.09$ for the unstable mode with the wave number $k=0.39$ in qualitative agreement with the numerically found value. It is worth noting that we have here the number of wavelengths along the ring of the soliton equal to $R k \cong 12$ in agreement with the number vortices formed at the nonlinear stage of development of the instability. Thus, the snake instability theory agrees at least qualitatively with the process observed in numerical simulations.

\section{Conclusion}

The idea that bright solitons behave as "particles" goes back to the beginning of modern nonlinear physics. However, it was not so obvious that it could be applied to dark solitons since their behavior depends also on the background evolution. Konotop and Pitaevskii noticed [4 that if background is a stationary solution of the GP equation in the TF approximation, then dynamics of dark solitons is determined by conservation of their energy. We have shown in this Letter that this idea can be extended to dynamics of ring solitons. This simple 

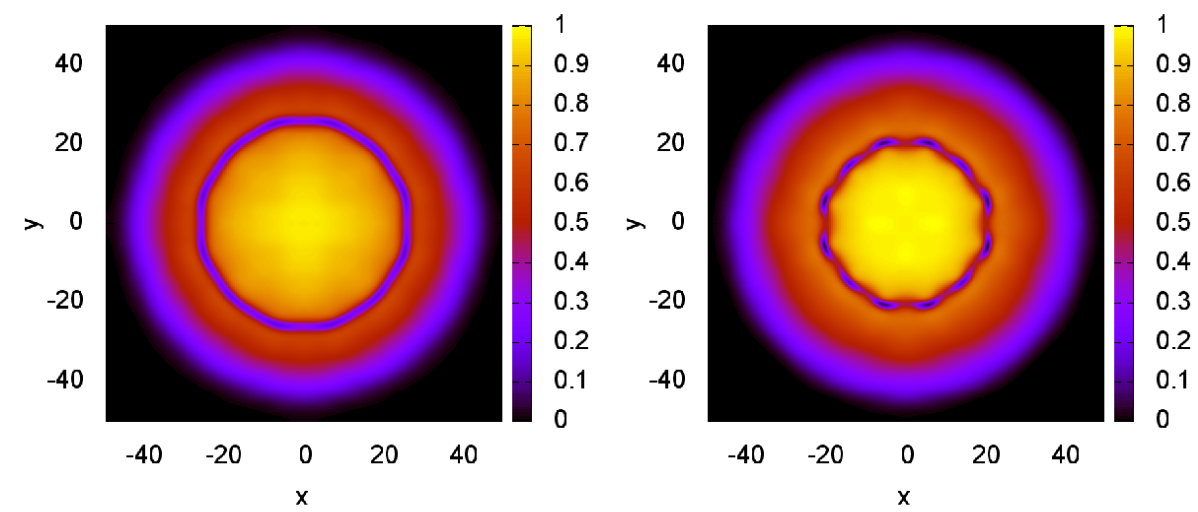

Figure 3: Transformation of a ring dark soliton into necklace of vortices: density plots at the moments of time (a) $t=120$ with a slightly disturbed dark soliton and (b) $t=130$ with well developed necklace of vortices.

approach can be easily generalized to arbitrary form of the nonlinear repulsive self-action what is important for applications to nonlinear optics. Examples discussed in this Letter show that this approach gives accurate enough description of motion of ring dark solitons up to the moment of their decay to vortices due to snake instability.

\section{Acknowledgments}

We are grateful to Yu.G. Gladush and L.A. Smirnov for useful discussions. This work was supported by RFBR (grant 09-02-00499). We thank also Joint Supercomputer Center of RAS for the provision of computing facilities.

\section{References}

[1] Y.S. Kivshar and B. Luther-Davies, Phys. Rep. 298 (1998) 81.

[2] D.J. Frantzeskakis, J. Phys. A: Math. Theor. 43 (2010) 213001.

[3] Th. Busch and J.R. Anglin, Phys. Rev. Lett. 84 (2000) 2298.

[4] V.V. Konotop and L.P. Pitaevskii, Phys. Rev. Lett. 93 (2004) 240403.

[5] V.A. Brazhnyi, V.V. Konotop and L.P. Pitaevskii, Phys. Rev. A 73 (2006) 053601 .

[6] A.M. Kamchatnov and M. Salerno, J. Phys. B: At. Mol. Opt. Phys. 42 (2009) 185303.

[7] Y.S. Kivshar and W. Królikowski, Optics Commun. 114 (1995) 353. 


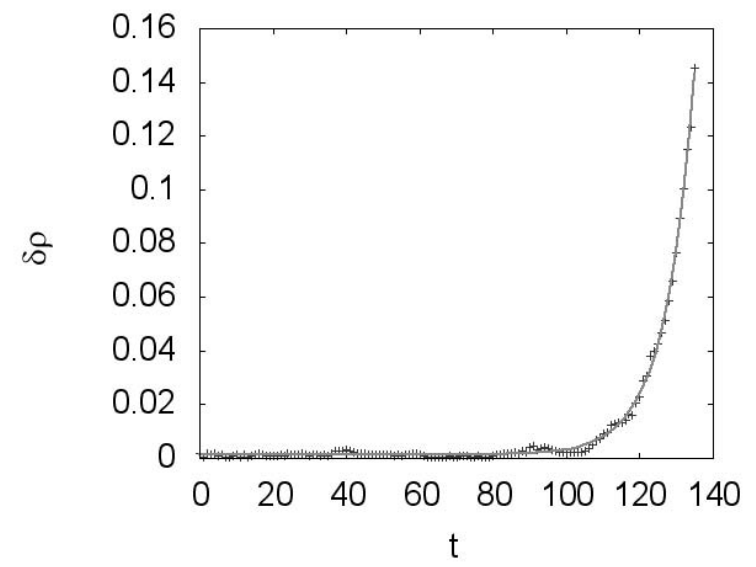

Figure 4: Dependence of the variable $\delta \rho$ on time: values from numerical solution of the GP equation are shown by crosses; a dashed line is a fitting curve 35 .

[8] Y.S. Kivshar and X. Yang, Phys. Rev. E 50 (1994) R40.

[9] A. Dreischuh, D. Neshev, G.G. Paulus, F. Grasbon, and H. Walther, Phys. Rev. E 66 (2002) 066611.

[10] G. Theocharis, D.J. Frantzeskakis, P.G. Kevrekidis, B.A. Malomed, and Y.S. Kivshar, Phys. Rev. Lett. 90 (2003) 120403.

[11] G. Theocharis, P. Schmelcher, M.K. Oberthaler, P.G. Kevrekidis, and D.J. Frantzeskakis, Phys. Rev. A 72 (2005) 023609.

[12] V.A. Mironov, A.I. Smirnov, and L.A. Smirnov, Zh. Eksp. Teor. Fiz. (2010) (in press).

[13] E.A. Kuznetsov and S.K. Turitsyn, Zh. Eksp. Teor. Fiz. 94 (1988) 119 [Sov. Phys. JETP, 67 (1988) 1583]. 